\title{
Renin-angiotensin system activation accelerates atherosclerosis in experimental renal failure by promoting endoplasmic reticulum stress-related inflammation
}

\author{
JIA YANG $^{1}$, XI ZHANG $^{1}$, XINYI YU $^{1}$, WEIXUE TANG $^{2}$ and HUA GAN ${ }^{1}$ \\ ${ }^{1}$ Department of Nephrology and ${ }^{2}$ Experimental Study Center, The First Affiliated Hospital of \\ Chongqing Medical University, Chongqing 400016, P.R. China
}

Received February 18, 2016; Accepted December 5, 2016

DOI: $10.3892 /$ ijmm.2017.2856

\begin{abstract}
In this study, we investigated the association between the renin-angiotensin system (RAS), endoplasmic reticulum (ER) stress and atherosclerosis (AS) in uremic apolipoprotein $\mathrm{E}$ knockout (apoE $\left.\mathrm{E}^{-/}\right)$mice. Mild uremia was induced by a $5 / 6$ nephrectomy $(5 / 6 \mathrm{Nx})$ in 10 -week-old apoE $\mathrm{E}^{-/-}$mice. Four weeks after nephrectomy, the mice received losartan or no treatment for 16 weeks. Sham-operated mice served as the controls. We found that uremia accelerated AS at the aortic root. The activation of ER stress and the significant upregulation of pro-inflammatory cytokines and chemokines were observed in the uremic mice. Phosphorylated inositol-requiring $1 \alpha$ (p-IRE1 $\alpha$ ), an ER stress marker protein, was mainly expressed in macrophages in the atherosclerotic lesions. Treatment with losartan significantly attenuated aortic AS, inhibited ER stress and reduced aortic inflammation. In in vitro experiments, angiotensin II (Ang II) increased the levels of the common ER stress maker, glucoseregulated protein 78 (GRP78) and the phosphorylation of IRE1 $\alpha$ in RAW264.7 macrophages. Treatment with losartan inhibited the activation of ER stress and the upregulation of GRP78, and enhanced the expression of nuclear factor- $\kappa \mathrm{B}(\mathrm{NF}-\kappa \mathrm{B})$ inhibitor (IкB) in Ang II-stimulated RAW264.7 macrophages. IRE1 $\alpha$-siRNA suppressed inflammation and downregulated I $\kappa$ B expression and I $\kappa$ B kinase (IKK) phosphorylation, which inhibited $\mathrm{I} \kappa \mathrm{B}$ degradation and NF- $\mathrm{NB}$ p65 nuclear translocation in Ang II-treated RAW264.7 macrophages. These findings suggest that RAS activation accelerates AS by promoting ER stress-related inflammation in uremic mice.
\end{abstract}

\section{Introduction}

Patients with chronic renal failure (CRF) have a greater risk of developing cardiovascular disease (CVD) than subjects with

Correspondence to: Professor Hua Gan, Department of Nephrology, The First Affiliated Hospital of Chongqing Medical University, 1 Yixueyuan Road, Yuzhong, Chongqing 400016, P.R. China

E-mail: ganhuadr@163.com

Key words: uremia, atherosclerosis, angiotensin II, endoplasmic reticulum stress, inflammation normal renal function. Atherosclerosis (AS) is an important risk factor for the development of CVD in patients with CRF (1-4). Previous studies have reported that inflammation plays a key role in the development of AS in patients with CRF (5-7).

The endoplasmic reticulum (ER) is a multifunctional organelle that performs protein synthesis, folding and maturation. An accumulation of unfolded or misfolded proteins in the ER lumen leads to ER stress, which restores homeostasis in the ER. The release of ER stress sensors, including inositolrequiring $1 \alpha($ IRE $1 \alpha)$, double-stranded RNA-dependent protein kinase (PKR)-like ER kinase (PERK) and activating transcription factor 6 (ATF6) stimulates three distinct downstream signaling pathways $(8,9)$. Severe and prolonged ER stress disrupts homeostasis and causes tissue injury and organ dysfunction. ER stress has been implicated in various types of CVD. Increasing evidence indicates that ER stress is the upstream signal for the inflammatory reaction, and is thus a key player in AS initiation and progression (10-13).

As regards other diseases, the suppression of the reninangiotensin system (RAS) can also inhibit ER stress to decrease angiotensin II (Ang II)-induced inflammation (14,15). Thus, RAS activation promotes ER stress-related inflammation and may aggravate AS and other diseases. According to previous studies, RAS inhibition effectively abolishes the pro-atherogenic effects of uremia, and these effect are mainly dependent on an anti-inflammatory mechanism $(16,17)$. However, the association between RAS-mediated ER stress and uremia-associated AS has not yet been examined to date, at least to the best of our knowledge. In this study, we investigated the effects of RAS on ER stress-induced inflammation in uremic apolipoprotein $\mathrm{E}$ knockout $\left(\mathrm{apoE}^{--}\right.$) mice by blocking the Ang II receptor to further clarify the mechanisms of action of RAS in experimental uremiaassociated AS.

\section{Materials and methods}

Serum biochemistry. Serum levels of urea nitrogen (BUN) and creatinine (CRE) were measured enzymatically with reagents from Nanjing Jiancheng Bioengineer Institute (Nanjing, China). The serum level of Ang II was measured by ELISA according to the instructions provided by the manufacturer (Jianglai Biotechnology Co., Shanghai, China). 
Animal models. Ten-week-old male apoE $\mathrm{E}^{-/-}$mice (homozygous apoE-deficient male mice, back-crossed 20 times from the C57BL/6 strain; Vital River Laboratory Animal Technology Co., Ltd., Beijing, China) were housed in the animal facility of Chongqing Medical University, Chongqing, China. The animals were maintained in an air-conditioned, pathogen-free and light-controlled environment and were fed standard mouse chow (2018S; Harlan Teklad, Madison, WI, USA) and sterile water. Experimental mild uremia was induced by $5 / 6$ nephrectomy $(5 / 6 \mathrm{Nx}$, termed $\mathrm{SNx})$. In total, $31 \mathrm{apoE}^{-1-}$ mice were randomly allocated to undergo $\mathrm{SNx}(\mathrm{n}=21)$ or sham operation $(n=10)$. At 10 weeks of age, both the upper poles of the right kidney were resected, and electrocoagulation of the incision was performed to stop bleeding. After 2 weeks, the whole left kidney was removed. The control animals underwent a sham operation (the bilateral kidneys were only exposed, without any resection of the kidneys). Anesthesia was achieved by an intraperitoneal injection of sodium pentobarbital (Sigma Chemical Co., St. Louis, MO, USA) at a dose of $40 \mathrm{mg} / \mathrm{kg}$.

Four weeks following nephrectomy, the uremic mice were randomly allocated into 2 subgroups as follows: the mice treated with the Ang II type 1 (AT1) receptor antagonist, losartan (Merck Sharp \& Dohme Pty. Ltd., Macquarie Park, $\mathrm{NSW}$, Australia) at a dose of $30 \mathrm{mg} / \mathrm{kg}$ in drinking water $(\mathrm{n}=10)$ or no medicine $(\mathrm{n}=11)$ for 12 weeks. The sham-operated mice received no medicine $(\mathrm{n}=10)$.

After the 12-week-intervention, each mouse was anesthetized, and blood was collected from the retro-orbital venous plexus. The blood was centrifuged at $3,000 \mathrm{rpm}$, at $4^{\circ} \mathrm{C}$, and the plasma was stored at $-80^{\circ} \mathrm{C}$ for analysis. For each mouse, following perfusion, the heart and aorta were separated from the iliac arteries. The heart and aortic root were dissected from the distal aorta and stored in 4\% paraformaldehyde solution for histological analyses. The remaining aorta was stored at $-80^{\circ} \mathrm{C}$ for gene and protein evaluations of the atherosclerotic lesions. This study was performed in strict accordance with the recommendations in the Guide for the Care and Use of Laboratory Animals of Chongqing Medical University. The protocols were approved by the Institutional Ethics Committee for Animal Experiments of Chongqing Medical University.

Cell culture and treatment. RAW264.7 murine macrophages were obtained from the Type Culture Collection of the Chinese Academy of Sciences (Shanghai, China) and grown in Dulbecco's modified Eagle's medium (DMEM) containing 10\% fetal bovine serum (FBS) (both from HyClone, Logan, UT, USA). The cells were plated on tissue culture dishes before the experiment and were cultured at $37^{\circ} \mathrm{C}$ under $5 \% \mathrm{CO}_{2}$ in a humidified incubator. In all the experiments, the RAW264.7 macrophages were grown to $90 \%$ confluence and were serum-starved for $12 \mathrm{~h}$ prior to exposure to to various concentrations of Ang II $(0.01,0,1,1$, or $10 \mu \mathrm{g} / \mathrm{ml}$ ). Prior to exposure to $1 \mu \mathrm{g} / \mathrm{ml}$ Ang II for $8 \mathrm{~h}$, the cells were treated with losartan $(10 \mu \mathrm{mol} / \mathrm{l}) 1 \mathrm{~h}$ before.

Infection with lentivirus. To verify whether Ang II-induced inflammation is associated with ER stress, the RAW264.7 macrophages were transfected with a specific siRNA-carrying lentiviral vector directed against IRE1 $\alpha$ (forward, 5'-GGAA UUACUGGCUUCUCAUdTdT-3' and reverse, 5'-AUGAGAA GCCAGUAAUUCCdTdT-3') (18). A nonsense sequence served
Table I. Sequences of primers for the detected genes.

\begin{tabular}{ll}
\hline Gene & \multicolumn{1}{c}{ Primer sequences $\left(5^{\prime} \rightarrow 3^{\prime}\right)$} \\
\hline IL-6 & F: GAGGATACCACTCCCAACAGACC \\
& R: AAGTGCATCATCGTTGTTCATACA \\
TNF- $\alpha$ & F: CATGAGCACAGAAAGCATGATCCG \\
& R: AAGCAGGAATGAGAAGAGGCTGAG \\
CCL2/MCP-1 & F: CTTCCTCCACCACCATGCA \\
& R: CCAGCCGGCAACTGTGA \\
CX3CL1 & F: GTGCTGACCCGAAGGAGAAA \\
& R: CACCCGCTTCTCAAACTTGC \\
GADPH & F: TGCTGAGTATGTCGTGGAGTCTA \\
& R: AGTGGGAGTTGCTGTTGAAATC
\end{tabular}

F, forward; R, reverse; IL-6, interleukin-6; TNF- $\alpha$, tumor necrosis factor- $\alpha$; CCL2, chemokine (C-C motif) ligand 2; MCP-1, monocyte chemoattractant protein-1; CX3CL1, chemokine (C-X3-C motif) ligand 1; GADPH, glyceraldehyde 3-phosphate dehydrogenase.

as the negative control. Following transfection for $48 \mathrm{~h}$, the cells were exposed to Ang II for $8 \mathrm{~h}$. Target gene silencing was validated by reverse transcription-quantitative PCR (PCR).

Histopathological analysis. Following fixation, the hearts and aortic roots were paraffin-embedded and sectioned. The sections were stained with hematoxylin-eosin or special antibodies, respectively. For immunohistochemistry, paraffinized sections were deparaffinized.Endogenous peroxidase activity was blocked with $3 \% \mathrm{H}_{2} \mathrm{O}_{2}$ for $20 \mathrm{~min}$, and non-specific staining was blocked by incubation with goat serum for $15 \mathrm{~min}$. The samples were stained with anti-CD68 (BA3638; 1:100; Boster, Wuhan, China) or anti-p-IRE1 $\alpha$ (Ser724; ab48187; 1:300; Abcam, Cambridge, UK) antibodies overnight at $4^{\circ} \mathrm{C}$, followed by HRP-conjugated secondary antibodies (SP-9001; ZSGB-Bio, Beijing, China) at a 1:200 dilution for $30 \mathrm{~min}$ at $37^{\circ} \mathrm{C}$. Immunohistochemical staining was performed by exposure to 3,3'-diaminobenzidine, and counterstaining was developed with hematoxylin. The sections were examined under a light microscope (Leica, Wetzlar, Germany) and quantified using Image-Pro Plus 6.0 software.

$R T-q P C R$. Total RNA was extracted from the rat aorta tissues or RAW264.7 macrophages using TRIzol reagent according to the manufacturer's instructions (Takara Bio, Inc., Otsu, Japan). RNA was reverse transcribed using the PrimeScript ${ }^{\mathrm{TM}} \mathrm{RT}$ reagent kit (Takara Bio, Inc.). qPCR was performed using an Applied Biosystems StepOnePlus Real-Time PCR system and $\mathrm{SYBR}^{\circledR}$-Green PCR master mix (both from Applied Biosystems, Foster City, CA, USA). Glyceraldehyde 3-phosphate dehydrogenase (GADPH) was used as an internal quantitative control. All cDNA were run twice in separate runs. The relative mRNA expression of each molecule was calculated using the $2^{-\Delta \Delta \mathrm{Ct}}$ method and normalized to GADPH. The primer sequences are listed in Table I.

Western blot analysis. The aorta tissue or RAW264.7 macrophages were lysed for $30 \mathrm{~min}$ at $4^{\circ} \mathrm{C}$ in lysis buffer. Total protein concentrations were determined using bicinchoninic acid 
A

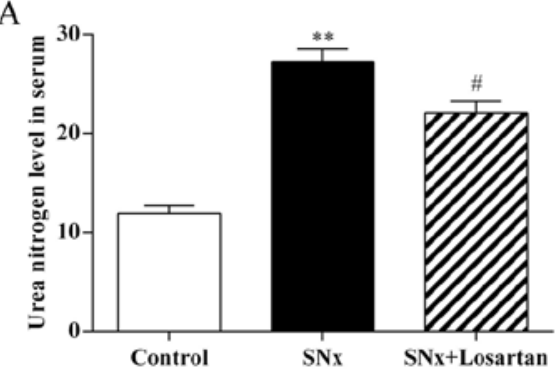

D

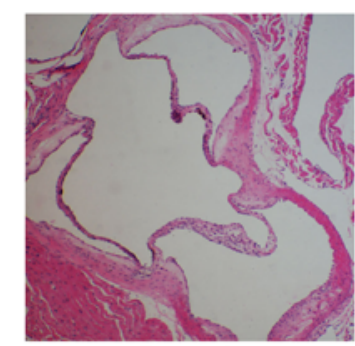

Control
B
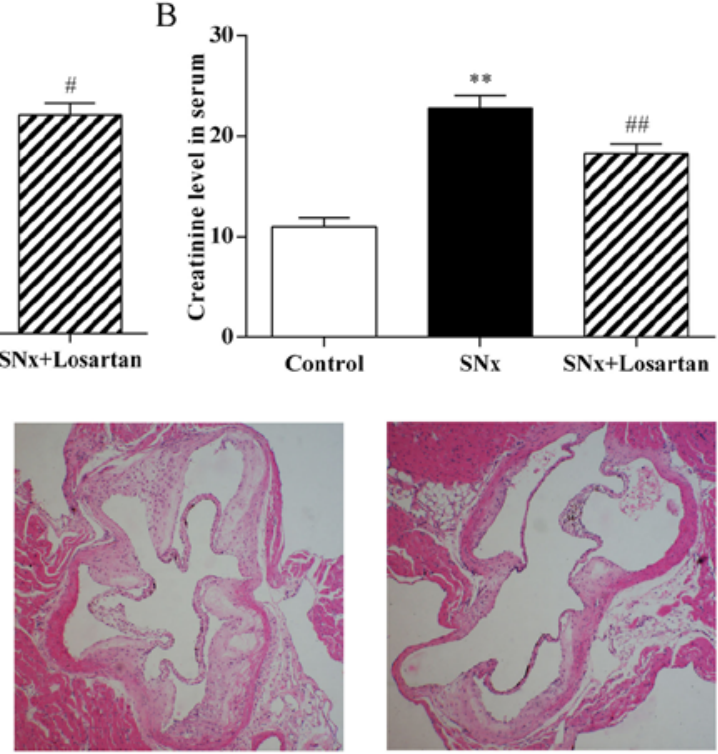

$\mathrm{SNx}$

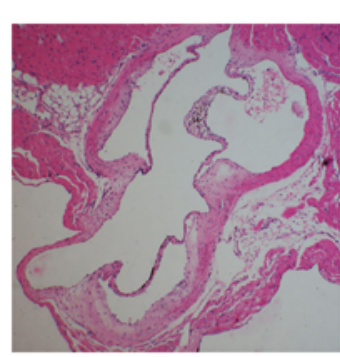

$\mathrm{SNx}+$ Losartan
C
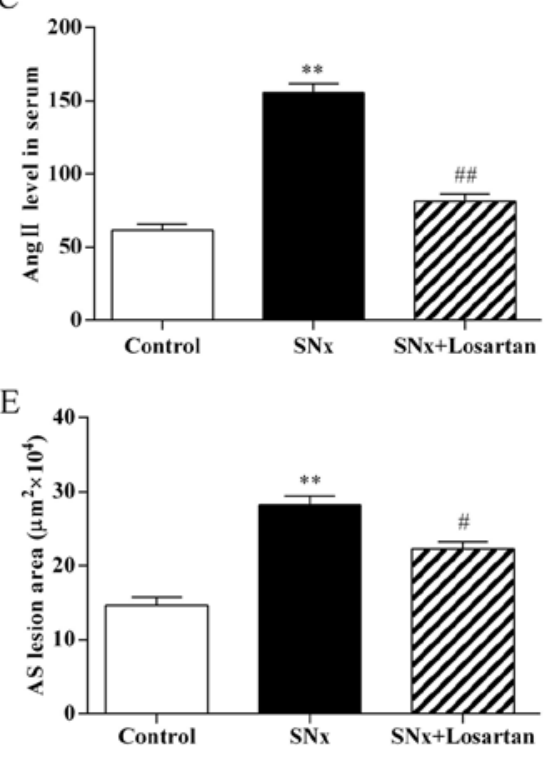

Figure 1. Effects of losartan treatment on serum biochemistry and the development of atherosclerosis in uremic mice. (A-C) Quantitative analysis of the level of urea nitrogen, creatinine and angiotensin II (Ang II) in serum. n=10, 11, 10 for the control, SNx and SNx + losartan groups, respectively. (D and E) Quantitative analysis of aortic root AS lesion area. Hematoxylin and eosin staining; original magnification, $\mathrm{x} 40 . \mathrm{n}=10,11,10$ for the control, SNx and SNx + losartan groups, respectively. SNx, subtotal nephrectomized apolipoprotein E knockout (apoE ${ }^{-/-}$) mice. Losartan was administered at the doses of $30 \mathrm{mg} / \mathrm{kg}$ for 12 weeks from 4 weeks after SNx. Data are the means \pm SEM. ${ }^{* *} \mathrm{P}<0.01$ vs. control group; ${ }^{\#} \mathrm{P}<0.05$ and ${ }^{\# \prime} \mathrm{P}<0.01$ vs. SNx group.

reagent. Total protein $(50-100 \mu \mathrm{g})$ was separated by sodium dodecyl sulfate-polyacrylamide gel electrophoresis (SDS-PAGE) and then transferred onto polyvinylidene difluoride membranes. After blocking, the membranes were incubated with antiIRE1 $\alpha$ (ab37073; 1:800; Abcam), anti-glucose-regulated protein 78 (GRP78; 11587-1-AP; 1:500; ProteinTech, Wuhan, China), anti-p-IRE1 $\alpha$ (Ser724; ab48187; 1:800; Abcam), IкB kinase $\alpha$ (IKK $\alpha$; 2682S; Cell Signaling Technology, Danvers, MA, USA), IкB kinase $\beta$ (IKK $\beta$; 2684S; Cell Signaling Technology), anti-p-IкB kinase $\alpha / \beta$ (p-IKK; Ser176/180; 2697T; 1:800; Cell Signaling Technology), IкB (YM3718; 1:500; ImmunoWay, Newark, DE, USA), anti-NF-кB p65 (YT5339; 1:500; ImmunoWay), anti- $\beta$-actin (6008-1-Ig; 1:750; ProteinTech) and anti-LaminB (sc-6216; 1:500; ZSGB-Bio) primary antibodies overnight at $4^{\circ} \mathrm{C}$. Horseradish peroxidase-conjugated secondary antibodies (ZB-2301; ZSGB-Bio) were applied the following day for $2 \mathrm{~h}$ at $37^{\circ} \mathrm{C}$. The bands were visualized using chemiluminescence (Beyotime, Shanghai, China). Band density was analyzed using fusion software (Bio-Rad Laboratories, Inc., Hercules, CA, USA) and normalized to $\beta$-actin or Lamin B density.

Statistical analyses. Statistical analyses were performed using SPSS software version 19.0. The data are presented as the means \pm SEM for in vivo experiments and as the means \pm SD for in vitro experiments. Analyses of variance (one-way ANOVA) followed by Bonferroni's or Dunnett's tests and the Kruskal-Wallis test followed by post-hoc tests were performed to evaluate group differences.

\section{Results}

Effect of losartan treatment on serum biochemistry and the development of AS in uremic mice. As shown in Fig. 1, 16 weeks after nephrectomy, the serum BUN and CRE concentrations were markedly increased in the uremic mice compared to the control mice $(\mathrm{P}<0.01$; Fig. $1 \mathrm{~A}$ and $\mathrm{B})$. These increases indicated that the model was successfully developed. In addition, the serum Ang II levels was significantly increased in the uremic mice compared to the controls $(\mathrm{P}<0.01$; Fig. 1C). Treatment with losartan treatment improved renal function in the uremic mice (BUN, P<0.05; CRE, P<0.01; Fig. 1A and B). The level of Ang II was also decreased in the losartan treatment group $(\mathrm{P}<0.01$; Fig. 1C). At 16 weeks after nephrectomy, the uremic mice displayed a significant increase in the aortic root plaque area compared with the control mice $(282,293 \pm 12,375$ vs. $146,627 \pm 10,411 \mu \mathrm{m}^{2}, \mathrm{P}<0.01$ ) (Fig. $1 \mathrm{D}$ and E). Treatment with losartan treatment markedly decreased the lesion area in the uremic mice $(222,703 \pm 9,857, \mathrm{P}<0.05)$ (Fig. 1D and E). These results indicate RAS inhibition ameliorated the development of accelerated AS in uremia mice.

Effect of losartan treatment on vascular inflammation and ER stress in uremic mice. As shown in Fig. 2, macrophage inflammation plays an important role in the development of AS and ER stress is the upstream signal for the inflammatory reaction. Using immunohistochemistry on adjacent sections, we stained macrophages with anti-CD68 antibodies and using p-IRE1 $\alpha$, a typical ER stress sensor, to evaluate the state of ER stress in the aortic root sections. The uremic mice had a significantly increased macrophage content within the aortic root plaques compared with the control mice $(29.64 \pm 1.59$ vs. $13.27 \pm 1.11 \%$, $\mathrm{P}<0.01$ ) (Fig. 2A and B). The uremic mice also exhibited a significant increase in p-IRE1 $\alpha$ expression $(24.40 \pm 1.28$ vs. $11.58 \pm 0.86 \%, \mathrm{P}<0.01$ ) (Fig. $2 \mathrm{C}$ and D). The co-localization of CD68 and p-IRE1 $\alpha$ on adjacent sections indicated that p-IRE1 $\alpha$ was mainly expressed in macrophages within the plaques. The results of western blot analysis revealed that the phosphorylation of IRE1 $\alpha$ was significantly increased in the aortas of the uremic 


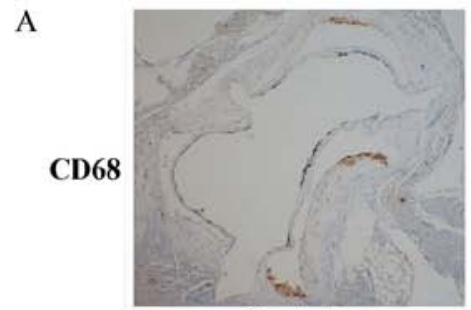

Control

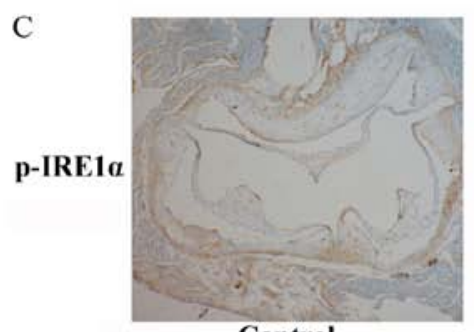

Control

E

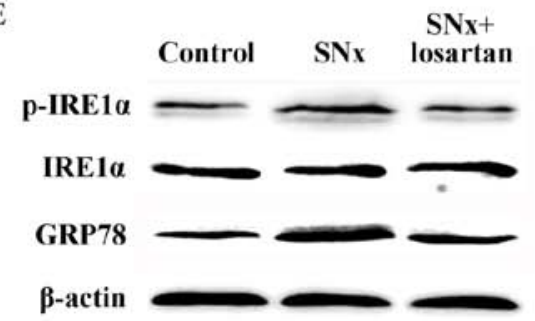

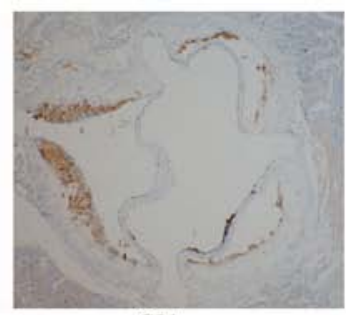

SNx

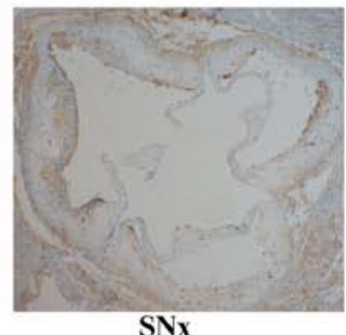

SNx
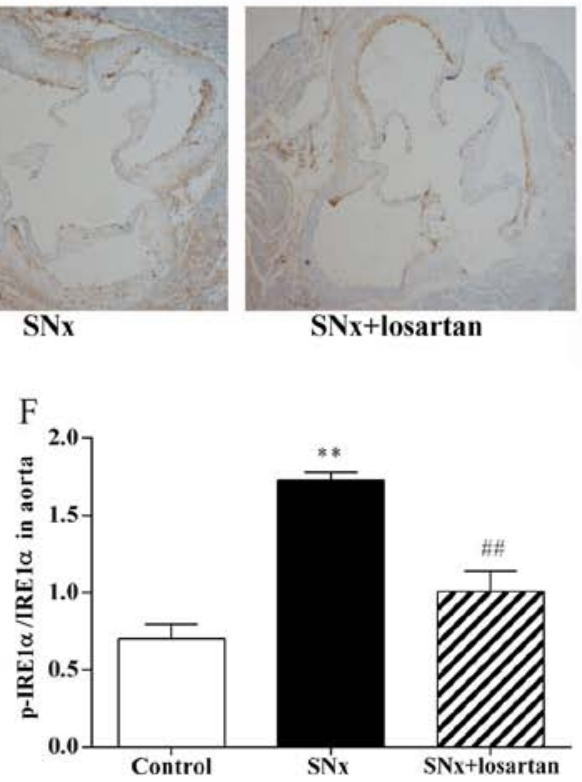

SNx+losartan

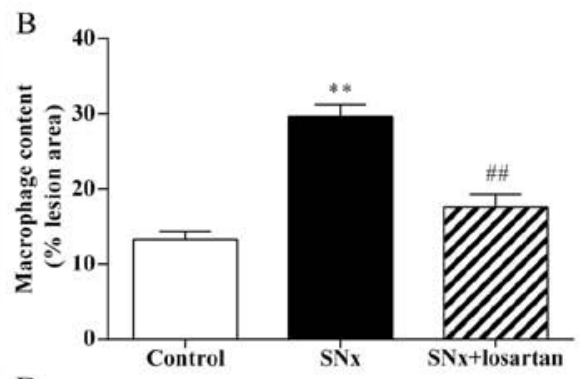

D

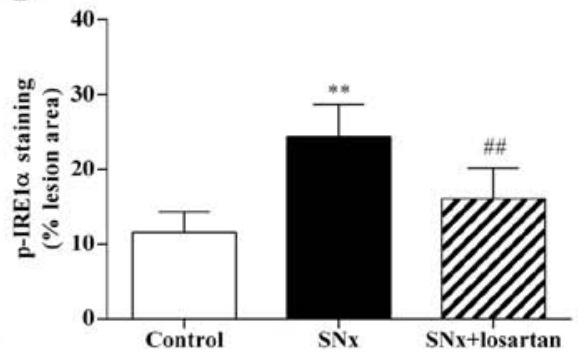

G

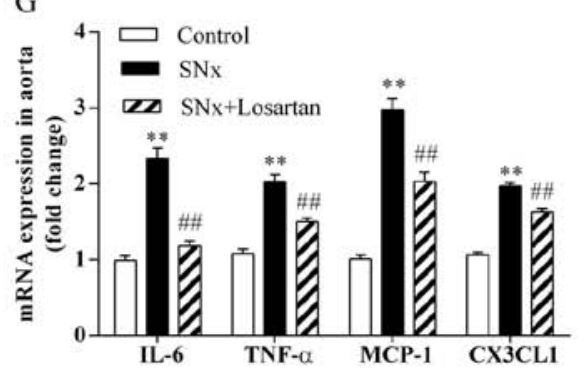

Figure 2. Effects of losartan treatment on vascular inflammation and ER stress in uremic mice. The co-localization of CD68 and p-inositol-requiring 1 $\alpha$ (IRE1 $\alpha$ ) was examined by immunohistochemistry using adjacent sections. (A and B) Quantitative analysis of macrophages content in lesion plaques. Immunohistochemistry with CD68, original magnification, $\mathrm{x} 40 . \mathrm{n}=10,11,10$ for the control, SNx and SNx + losartan groups, respectively. (C and D) Quantitative analysis of p-IRE1 $\alpha$ in lesion plaques in mice. Immunohistochemistry with p-IRE1 $\alpha$; original magnification, $\mathrm{x} 40$. (E and F) Western blot analysis of the activation of IRE1 $\alpha$ and the expression of glucose-regulated protein 78 (GRP78) in aortas of mice. n=5, 6, 5 for the control, SNx and SNx + losartan groups, respectively. (G) RT-qPCR analysis of pro-inflammatory cytokine and chemokine gene expression in aortas. $\mathrm{n}=5,5,5$ for the control, $\mathrm{SNx}$ and $\mathrm{SNx}+$ losartan groups, respectively. Data are the means \pm SEM. ${ }^{* *} \mathrm{P}<0.01$ vs. control group; ${ }^{\#} \mathrm{P}<0.01$ vs. SNx group.

mice $(\mathrm{P}<0.01)$ (Fig. 2E and F). Treatment with losartan significantly reduced the atherosclerotic plaque macrophage content in the uremic mice $(17.62 \pm 1.71 \%, \mathrm{P}<0.01)$ (Fig. 2A and $\mathrm{B}$ ). Treatment with losartan also markedly decreased $\mathrm{p}$-IRE1 $\alpha$ expression $(16.05 \pm 1.29, \mathrm{P}<0.01)$ (Fig. $2 \mathrm{C}$ and $\mathrm{D})$ and markedly inhibited IRE1 $\alpha$ phosphorylation in the uremic mice $(\mathrm{P}<0.01$; Fig. 2E and F). We also observed the upregulation of the common ER stress maker, GRP78, in the aortas of the uremic mice $(\mathrm{P}<0.01$; Fig. 2E). The upregulation of GRP78 was significantly inhibited in the aortas of the losartan-treated uremic mice $(\mathrm{P}<0.01$; Fig. $2 \mathrm{E})$. In addition, we compared the mRNA expression levels of pro-inflammatory cytokines, such as tumor necrosis factor- $\alpha$ (TNF- $\alpha$ ) and interleukin-6 (IL-6), and chemokines, such as chemokine (C-C motif) ligand 2 (CCL2)/monocyte chemoattractant protein-1 (MCP-1) and chemokine (C-X3-C motif) ligand 1 (CX3CL1), in the mouse aortas. The mRNA expression levels of the pro-inflammatory cytokines and chemokines were higher in the uremic mice than in the controls $(\mathrm{P}<0.01$; Fig. $2 \mathrm{G})$. Treatment with losartan markedly decreased the mRNA expression levels of the pro-inflammatory cytokines and chemokines in the uremic mice $(\mathrm{P}<0.05$; Fig. $2 \mathrm{G})$.
Effect of losartan on the expression of ER stress marker proteins and the $N F-\kappa B$ inhibitor protein I $\kappa B$ in Ang II-stimulated RAW264.7 macrophages. In this study, we examined the ER stress response in RAW264.7 macrophages following treatment with Ang II by measuring the protein levels of ER stress markers. Stimulation of the RAW264.7 macrophages with Ang II significantly increased the phosphorylation of IRE1 $\alpha$ in a time- and dose-dependent manner ( $\mathrm{P}<0.05$; Fig. 3A-D). Stimulation of the RAW264.7 macrophages with Ang II also significantly increased the GRP78 levels in a time- and dose-dependent manner $(\mathrm{P}<0.05$; Fig. 3E-H). Stimulation with Ang II also increased the phosphorylation of IKK $(\mathrm{P}<0.05$; Fig. $3 \mathrm{~K}$ and $\mathrm{L})$ in the RAW264.7 macrophages. Losartan is a common AT1 receptor antagonist. Losartan significantly inhibited the phosphorylation of IRE1 $\alpha$ and IKK $(\mathrm{P}<0.01$; Fig. 3I-L) compared with the Ang II-stimulated RAW264.7 macrophages not treated with losartan. $\mathrm{I} \kappa \mathrm{B}$ is a regulatory protein binding to the transcription factor, $\mathrm{NF}-\kappa \mathrm{B}$, retaining it in the cytoplasm. Losartan significantly enhanced the expression of I $\mathrm{B}$ in the Ang II-stimulated RAW264.7 macrophages ( $\mathrm{P}<0.01$; Fig. $3 \mathrm{~K}$ and $\mathrm{M}$ ), suggesting the inhibition of NF- $\mathrm{BB}$ by losartan in the Ang II-stimulated RAW264.7 macrophages. 

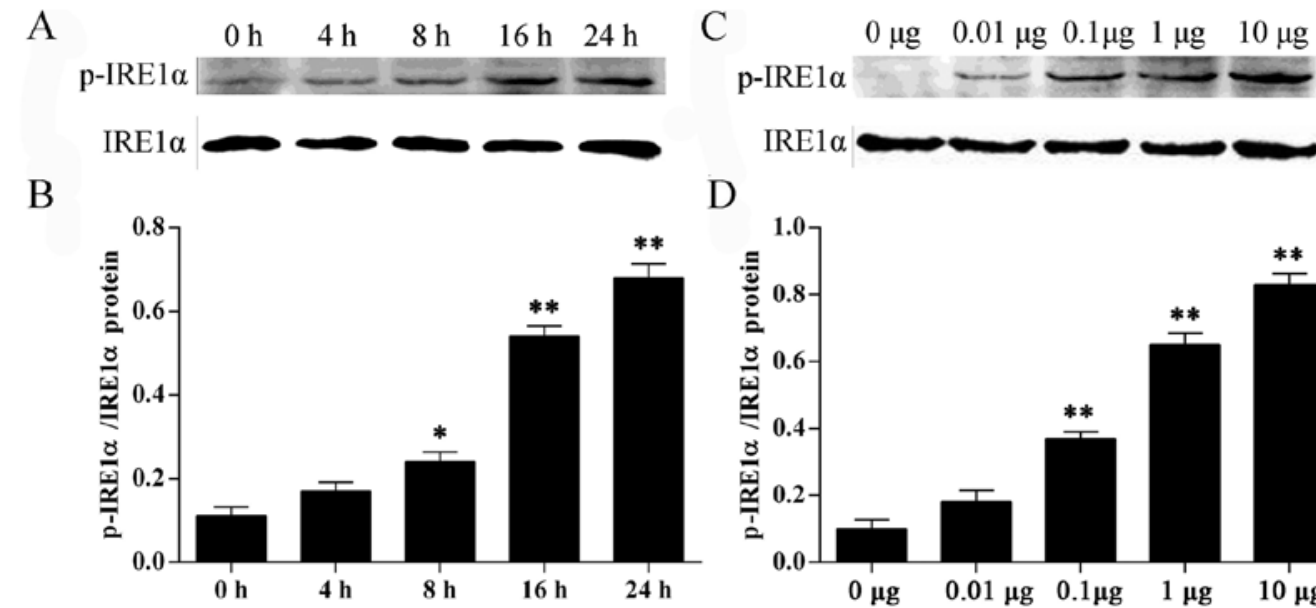

E

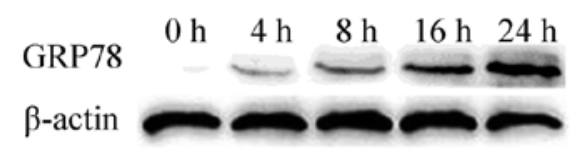

$\mathrm{F}$

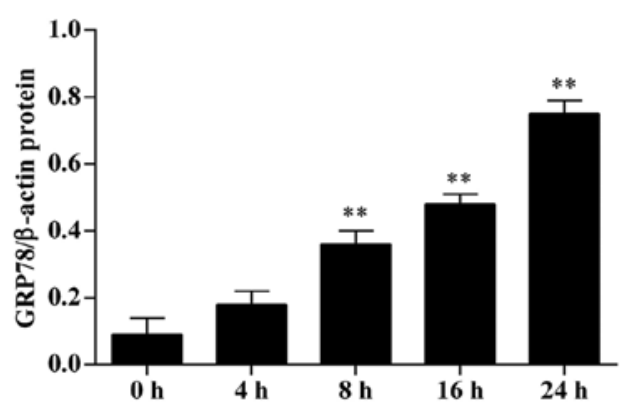

D

IRE1 $\alpha \longrightarrow$

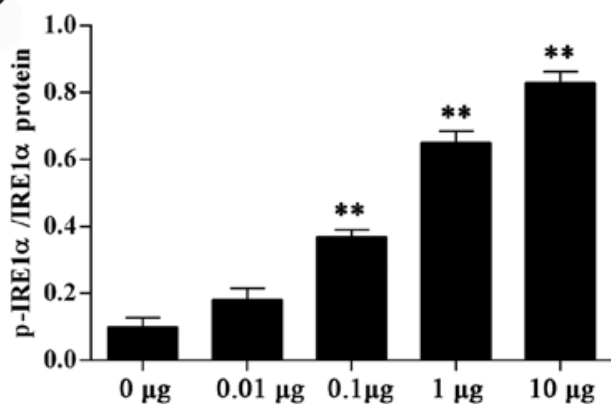

G

$0 \mu \mathrm{g} 0.01 \mu \mathrm{g} 0.1 \mu \mathrm{g} 1 \mu \mathrm{g} 10 \mu \mathrm{g}$

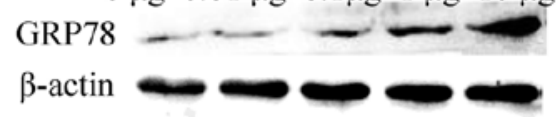

$\mathrm{H}$

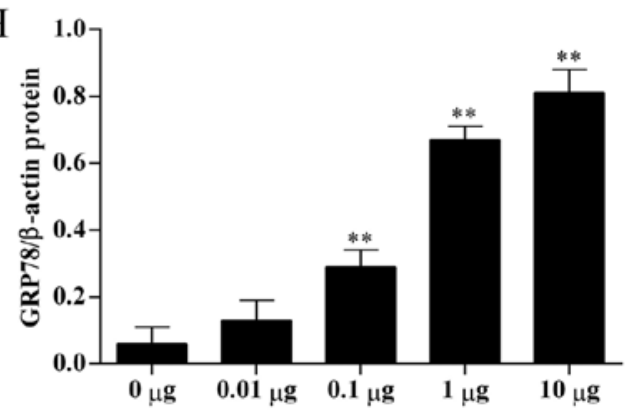

I

Control Losartan Ang II Ang II +Losartan

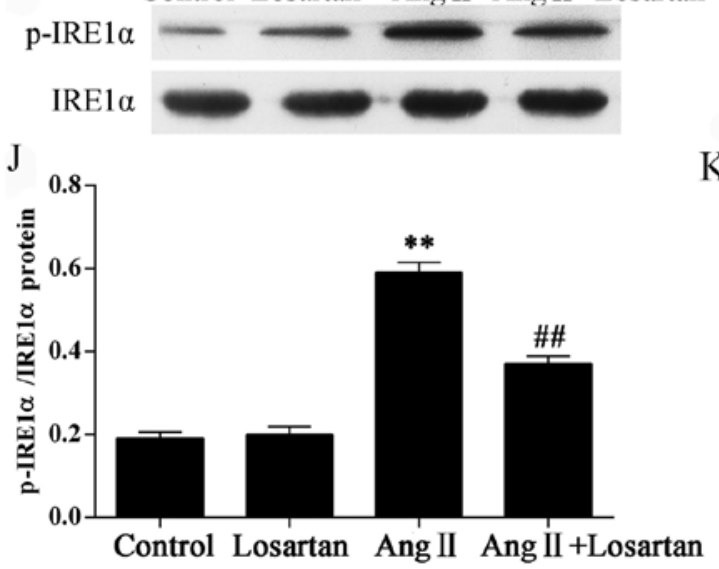

$\mathrm{L}$

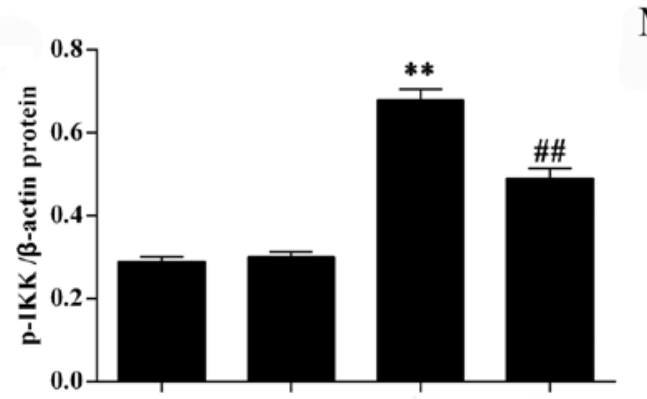

Control Losartan Ang II Ang II + Losartan

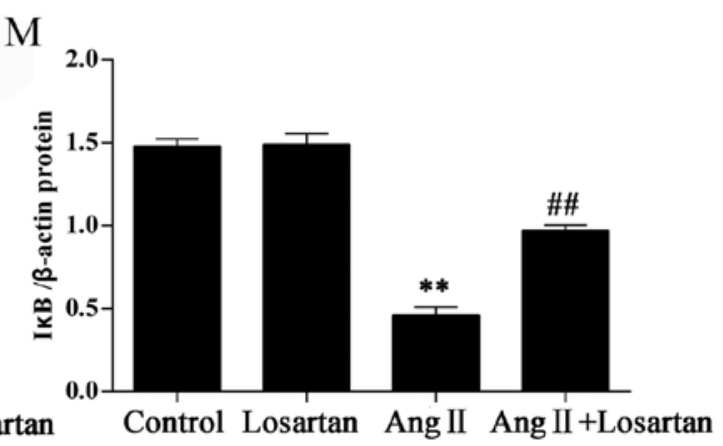

Figure 3. Effects of losartan on the expression of ER stress marker proteins and nuclear factor- $\kappa \mathrm{B}(\mathrm{NF}-\kappa \mathrm{B})$ inhibitor protein I $\kappa \mathrm{B}$ in angiotensin II (Ang II)stimulated RAW264.7 macrophages. RAW264.7 macrophages were stimulated with $1 \mu \mathrm{g} / \mathrm{ml}$ Ang II for $4,8,16$ and $24 \mathrm{~h}$ [for inositol-requiring $1 \alpha$ (IRE1 $\alpha$ ) activation (A and B); for glucose-regulated protein 78 (GRP78) (E and F)] or with 0.01, 0.1, 1, or $10 \mu \mathrm{g} / \mathrm{ml} \mathrm{Ang} \mathrm{II} \mathrm{for} 24 \mathrm{~h}$ [for IRE1 $\alpha$ activation (C and D); for GRP78 $(\mathrm{G}$ and $\mathrm{H})]$ and examined by western blot analysis. Culture medium was used as a blank control. (I-M) Western blot analysis of the activation of IRE1 $\alpha$, IKK $\alpha / \beta$ and IкB. The results were expressed as the means \pm SD. $n=3$ independent experiments; ${ }^{*} \mathrm{P}<0.05$ and ${ }^{* * *} \mathrm{P}<0.05$ vs. control; ${ }^{\# \#} \mathrm{P}<0.05$ vs. Ang II. 

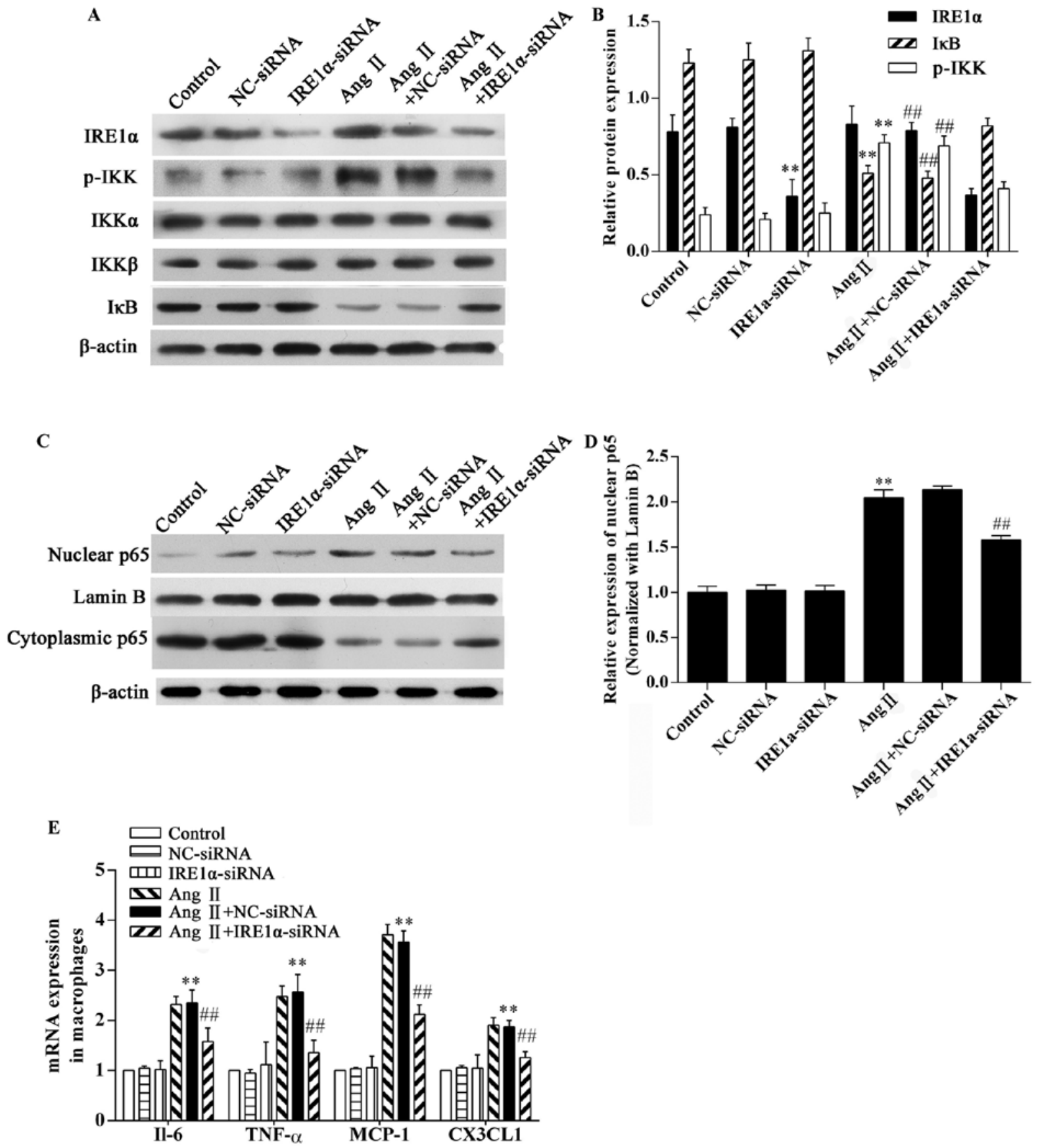

Figure 4. Effects of inositol-requiring $1 \alpha$ (IRE1 $\alpha$ ) siRNA on the angiotensin II (Ang II)-induced inflammatory response in RAW264.7 macrophages. The RAW264.7 macrophages transfected with or without IRE1 $\alpha$ siRNA were stimulated with $1 \mu \mathrm{g} / \mathrm{ml}$ Ang II for $8 \mathrm{~h}$ and then subjected to western blot analysis and gene expression analysis. Western blot analysis of the expression of (A and B) IRE1 $\alpha, \mathrm{p}-\mathrm{IKK}, \mathrm{IKK} \alpha / \beta, \mathrm{I} \mathrm{B}$, and (C and D) NF- $\mathrm{kB}$ p65. (E) RT-qPCR analysis of the gene expression of the indicated cytokines. The results were expressed as the mean $\pm \mathrm{SD}$. $\mathrm{n}=3$ independent experiments; ${ }^{* *} \mathrm{P}<0.05$ vs. control; ${ }^{\# \#} \mathrm{P}<0.05 \mathrm{vs}$. Ang II.

Effect of IREI $\alpha$-siRNA on the Ang II-induced inflammatory response in RAW264.7 macrophages. To confirm the role of ER stress in Ang II-induced inflammation, RAW264.7 macrophages were transfected with IREl $\alpha$-specific siRNA or control-siRNA $48 \mathrm{~h}$ prior to exposure to Ang II. The expression of the ER stress sensor, IRE1 $\alpha$, was downregulated in the IRE1 $\alpha$-siRNA group (Fig. 4A and B). The results of western blot analysis demonstrated that stimulation with Ang II significantly increased IKK phosphorylation $(\mathrm{P}<0.01$; Fig. $4 \mathrm{~A}$ and $\mathrm{B})$ and caused a marked degradation of IкB in the RAW264.7 macrophages ( $\mathrm{P}<0.01$; Fig. $4 \mathrm{~A}$ and $\mathrm{B})$. Ang II stimulation significantly increased NF- $\kappa \mathrm{B}$ p65 in nuclear extracts from macrophages, and the cytoplasmic NF-кB p65 content decreased (Fig. 4C). Compared with the Ang II-exposed group, IRE1 $\alpha$-siRNA decreased IKK phosphorylation and inhibited IאB degradation $(\mathrm{P}<0.01$; Fig. $4 \mathrm{~A}$ and $\mathrm{B})$ and inhibited NF- $\mathrm{\kappa B}$ p65 nuclear translocation ( $\mathrm{P}<0.01$; Fig. $4 \mathrm{C}$ and $\mathrm{D})$. In addition, Ang II stimulation significantly upregulated the mRNA expression of pro-inflammatory cytokines and chemokines in the RAW264.7 macrophages $(\mathrm{P}<0.01$; Fig. 4E). The depletion of IRE1 $\alpha$ expression markedly downregulated the mRNA expression of pro-inflammatory cytokines and chemokines (Fig. 4E). Without the presence of Ang II, the IRE1 $\alpha$-specific siRNA did not affect

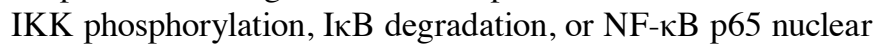


translocation (Fig. 4). The negative control (NC-siRNA) did not influence the effects of Ang II (Fig. 4). Thus, Ang II-mediated inflammation in RAW264.7 macrophages is partially dependent on the activation of ER stress.

\section{Discussion}

A number of experimental and clinical studies have demonstrated that RAS activation occurs during uremia, and RAS plays a key role in the development and progression of uremic AS $(16,17,19)$. Similar to these previous findings $(16)$, our study found that the Ang II serum concentration was significantly increased in uremic mice, and that RAS blockade with losartan significantly abolished the pro-atherogenic effects in apoE ${ }^{-/}$mice subjected to SNx. However, the exact mechanisms through which RAS promotes large vessel disease in patients with CRF were not fully explained.

Atherosclerotic plaques, particularly advanced lesions, contain abundant pro-inflammatory cytokines and chemokines $(20,21)$. Pedersen et al reported that the pro-inflammatory response overrules the pro-atherogenic potential of traditional factors during uremia-induced AS (6). In the present study, we induced experimental mild uremia by subjecting $\mathrm{apoE}^{-/-}$mice to SNx. The levels of Ang II were found to increase after SNx. It has been demonstrated that the anti-atherogenic effects of losartan are due to the direct inhibition of Ang II activity (22). Other events that occur due to Ang II, such as hypertension (15), may also be regulated by losartan. Furthermore, treatment with losartan has been shown to prevent both coronary vascular injury and myocyte damage induced by continuous Ang II infusion in rats (23). We aim to investigate the signaling pathways mediating the effects of losartan in the future.

Chronic inflammation is an important risk factor for the progression of AS in patients with CRF. According to our study, macrophage accumulation was increased, and pro-inflammatory cytokine and chemokine gene expression was upregulated in the aortas from uremic mice. Treatment with losartan, an AT1 receptor blocker, reduced macrophage accumulation and inhibited aortic inflammation in the uremic mice. These molecular and cellular changes were associated with accelerated AS. These findings are consistent with those of previous studies $(16,17)$. RAS inhibition abolished the proatherogenic effecta of uremia, which was mainly dependent on its anti-inflammatory mechanisms (16).

A recent study demonstrated that ER stress is a key player during the development of AS. Prolonged ER stress triggers macrophage and endothelial cell apoptosis, which in turn leads to plaque necrosis. Another important pro-atherogenic effect of prolonged ER stress is the activation of inflammatory pathways in cells within the plaques, especially macrophages (11). To determine the association between RAS and ER stress in uremic mice, we assessed the expression and activation of the ER stress response signaling proteins. GRP78 is a prominent ER-resident chaperone, which binds to the three ER stress sensors. During ER stress, GRP78 stably interacts with misfolded or unfolded proteins, and the three previously mentioned GRP78-bound proteins, PERK, IRE1 $\alpha$ and ATF6 are free. The upregulation of GRP78 is a common ER stress marker (10). In this study, we observed that GRP78 upregulation was significantly inhibited in the aortas of losartan-treated uremic mice. The three free proteins can activate their respective downstream signaling pathways, and prolong the activation of ER stress, causing disease. In this study, among the three branches of ER stress signaling, we focused on IRE1 $\alpha$ to address the inflammation caused by uremia. In addition, the effects of Ang II and losartan upon endothelial and vascular smooth cells in the pathogenesis of AS cannot be neglected. A previous in vivo study demonstrated that IRE1 signaling is essential for ischemia-induced vascular endothelial growth factor (VEGF)-A expression and contributes to angiogenesis (24). Exogenous Ang II can activate IRE1 protein expression in a dose-dependent manner in endothelial cells, and the activation of IRE1 is necessary for both VEGF production and capillary tube formation from endothelial cells induced by Ang II (25).

IRE1 $\alpha$ activates both the NF- $\mathrm{KB}$ and AP-1 transcriptional pathways, which are crucial regulators of the inflammatory response (26-29). It is widely accepted that IRE1 is involved in the activation of NF- $\mathrm{KB}$ induced by ER stress. In a previous study, the activation of NF- $\mathrm{KB}$ by the ER stress-inducing agents, thapsigargin and tunicamycin, was inhibited by a dominantnegative IRE1 (27). Our results also suggested that the NF- $\mathrm{kB}$ activation from the ER is transduced by IRE1. Moreover, the study by Miyazaki-Anzai et al demonstrated that CHOP inhibition alone was not sufficient to inhibit CKD-dependent AS. The IRE1 $\alpha-\mathrm{NF}-\kappa \mathrm{B}$ axis of the ER stress signal may be more important in the development of CKD-dependent AS (30). In this study, losartan treatment decreased IREl $\alpha$ activation in atherosclerotic plaques. From this perspective, we suggest that RAS activation may be associated with ER stress-induced inflammation during uremic AS.

To further explore the association between RAS activation and ER stress-related inflammation, we performed in vitro experiments. In the in vivo experiments, we discovered the co-localization of CD68 and p-IRE1 $\alpha$ by immunohistochemistry using adjacent sections, showing that p-IREl $\alpha$ was mainly expressed in macrophages within the plaques. ER stress-induced inflammation in macrophages is an important contributor to AS (11). During early development of AS, monocytes migrate into the arterial intima by chemotaxis and then mature into macrophages. Macrophage infiltration into the arteries is a key factor for atherosclerotic plaque initiation. Macrophages in plaques produce pro-inflammatory cytokines to promote AS progression $(31,32)$. In our in vitro experiment, Ang II was used to stimulate RAW264.7 macrophages as Ang II is the primary effector of RAS. The results demonstrated that ER stress was induced by Ang II stimulation in RAW264.7 macrophages. GRP78 expression was significantly upregulated, and IRE1 $\alpha$ phosphorylation was increased in the Ang II-stimulated macrophages. During ER stress, IRE1 $\alpha$ is activated by autophosphorylation at S724, and IRE1 $\alpha$ autophosphorylation induces a conformational change in its cytosolic domain, which can subsequently bind to the adaptor protein TNF- $\alpha$-receptor associated factor 2 (TRAF2). The IRE1 $\alpha$-TRAF2 complex can phosphorylate IKK, which leads to I $\kappa \mathrm{B}$ degradation and the nuclear translocation of NF- $\mathrm{BB}$ (26-28). In this study, we determined that stimulation with Ang II significantly upregulated IKK phosphorylation, I $\kappa \mathrm{B}$ degradation and NF- $\kappa \mathrm{B}$ nuclear translocation. More importantly, IRE1 $\alpha$ depletion effectively downregulated IKK phosphorylation and inhibited IкB degradation and NF- $\kappa B$ nuclear translocation. 
In previous studies, Ang II stimulation was shown to effectively activate an inflammatory response. Guo et al indicated that $N F-\kappa B$ is a critical inflammatory transcription factor for Ang II-induced inflammation in RAW264.7 macrophages (33). $\mathrm{NF}-\kappa \mathrm{B}$ is a pro-atherogenic factor, mainly due to its regulation of many pro-inflammatory genes that are linked to AS $(34,35)$. This study indicated that ER stress is an upstream signal of the NF- $\mathrm{B}$ pathway. Ang II stimulation significantly elevated pro-inflammatory cytokine and chemokine gene expression in RAW264.7 macrophages, which was mostly dependent on the activation of the IRE $\alpha / \mathrm{IKK} / \mathrm{NF}-\kappa \mathrm{B}$ pathway. In this study, losartan significantly enhanced the expression of I $\mathrm{B}$ in Ang II-stimulated RAW264.7 macrophages, suggesting the inhibition of NF- $\kappa$ B by losartan in Ang II-stimulated RAW264.7 macrophages. This further confirmed the effect of losartan on the IRE $\alpha / \mathrm{IKK} / \mathrm{NF}-\kappa \mathrm{B}$ pathway in vitro. In addition, it has been demonstrated that phosphorylated forms of IRE1 $\alpha$ could be detected by slower migration upon stimulation with lipopolysaccharide (36). The knockdown of IRE1 $\alpha$ in J774 macrophages resulted in reduced IL- 6 induction by tunicamycin and lipopolysaccharide co-treatment (36). Similarly, the effect of IRE1 $\alpha$-siRNA on the Ang II-induced inflammatory response is not only due to the reduced macrophage number.

Furthermore, MCP-1 has been detected in atherosclerotic lesions $(37,38)$. Blocking the expression of MCP-1 or its receptor CCR2 decreases atheroma formation in hypercholesterolemic mice $(39,40)$. In future studies we aim to explore the influence of MCP-1 and its receptor, CCR2, on ER stress-induced AS.

In conclusion, our findings demonstrated that the blockade of RAS by losartan almost completely prevented the acceleration of atherosclerotic lesion formation in uremic apoE ${ }^{-/-}$mice. This effect was associated with the modulation of chronic inflammation via ER stress-dependent mechanisms. This study further clarified the mechanism through which RAS aggravates and accelerates atherogenesis in uremic mice.

\section{Acknowledgements}

We are grateful to Mr. Zhengyi Wang, Ms. Jingmei Xie and Ms. Yao Xiao for providing experiment technical support.

\section{References}

1. Go AS, Chertow GM, Fan D, McCulloch CE and Hsu CY: Chronic kidney disease and the risks of death, cardiovascular events, and hospitalization. N Engl J Med 351: 1296-1305, 2004.

2. Amann K, Gross ML and Ritz E: Pathophysiology underlying accelerated atherogenesis in renal disease: Closing in on the target. J Am Soc Nephrol 15: 1664-1666, 2004.

3. Foley RN, Parfrey PS and Sarnak MJ: Clinical epidemiology of cardiovascular disease in chronic renal disease. Am J Kidney Dis 32 (Suppl 3): S112-S119, 1998.

4. Seliger SL, Gillen DL, Tirschwell D, Wasse H, Kestenbaum BR and Stehman-Breen CO: Risk factors for incident stroke among patients with end-stage renal disease. J Am Soc Nephrol 14: 2623-2631, 2003.

5. Banerjee D, Recio-Mayoral A, Chitalia N and Kaski JC: Insulin resistance, inflammation, and vascular disease in nondiabetic predialysis chronic kidney disease patients. Clin Cardiol 34: 360-365, 2011

6. Pedersen TX, Binder CJ, Fredrikson GN, Nilsson J, Bro S and Nielsen LB: The pro-inflammatory effect of uraemia overrules the anti-atherogenic potential of immunization with oxidized LDL in apoE ${ }^{--}$mice. Nephrol Dial Transplant 25: 2486-2491, 2010 .
7. Yilmaz MI, Stenvinkel P, Sonmez A, Saglam M, Yaman H, Kilic S, Eyileten T, Caglar K, Oguz Y, Vural A, et al: Vascular health, systemic inflammation and progressive reduction in kidney function; clinical determinants and impact on cardiovascular outcomes. Nephrol Dial Transplant 26: 3537-3543, 2011.

8. Pluquet O, Pourtier A and Abbadie C: The unfolded protein response and cellular senescence. A review in the theme: Cellular mechanisms of endoplasmic reticulum stress signaling in health and disease. Am J Physiol Cell Physiol 308: C415-C425, 2015.

9. Ron D and Walter P: Signal integration in the endoplasmic reticulum unfolded protein response. Nat Rev Mol Cell Biol 8: 519-529, 2007.

10. Sozen E, Karademir B and Ozer NK: Basic mechanisms in endoplasmic reticulum stress and relation to cardiovascular diseases. Free Radic Biol Med 78: 30-41, 2015.

11. Gotoh T, Endo M and Oike Y: Endoplasmic reticulum stress-related inflammation and cardiovascular diseases. Int J Inflamm 2011: 259462, 2011.

12. Tabas I: The role of endoplasmic reticulum stress in the progression of atherosclerosis. Circ Res 107: 839-850, 2010.

13. Zhou J, Lhoták S, Hilditch BA and Austin RC: Activation of the unfolded protein response occurs at all stages of atherosclerotic lesion development in apolipoprotein E-deficient mice. Circulation 111: 1814-1821, 2005.

14. Wang J, Wen Y, Lv LL, Liu H, Tang RN, Ma KL and Liu BC: Involvement of endoplasmic reticulum stress in angiotensin II-induced NLRP3 inflammasome activation in human renal proximal tubular cells in vitro. Acta Pharmacol Sin 36: 821-830, 2015.

15. Young CN, Li A, Dong FN, Horwath JA, Clark CG and Davisson RL: Endoplasmic reticulum and oxidant stress mediate nuclear factor- $\kappa \mathrm{B}$ activation in the subfornical organ during angiotensin II hypertension. Am J Physiol Cell Physiol 308: C803-C812, 2015.

16. Bernardi S, Candido R, Toffoli B, Carretta R and Fabris B: Prevention of accelerated atherosclerosis by AT1 receptor blockade in experimental renal failure. Nephrol Dial Transplant 26: 832-838, 2011.

17. Bro S, Binder CJ, Witztum JL, Olgaard K and Nielsen LB: Inhibition of the renin-angiotensin system abolishes the proatherogenic effect of uremia in apolipoprotein E-deficient mice. Arterioscler Thromb Vasc Biol 27: 1080-1086, 2007.

18. Yao S, Miao C, Tian H, Sang H, Yang N, Jiao P, Han J, Zong C and Qin S: Endoplasmic reticulum stress promotes macrophage-derived foam cell formation by up-regulating cluster of differentiation 36 (CD36) expression. J Biol Chen 289: 4032-4042, 2014.

19. Berger AK, Duval S and Krumholz HM: Aspirin, beta-blocker, and angiotensin-converting enzyme inhibitor therapy in patients with end-stage renal disease and an acute myocardial infarction. J Am Coll Cardiol 42: 201-208, 2003.

20. Mach F: The role of chemokines in atherosclerosis. Curr Atheroscler Rep 3: 243-251, 2001.

21. Hansson GK: Inflammatory mechanisms in atherosclerosis. J Thromb Haemost 7 (Suppl 1): 328-331, 2009.

22. Liang C, Wu ZG, Ding J, Jiang JF, Huang GZ, Du RZ and Ge JB: Losartan inhibited expression of matrix metalloproteinases in rat atherosclerotic lesions and angiotensin II-stimulated macrophages. Acta Pharmacol Sin 25: 1426-1432, 2004.

23. Kabour A, Henegar JR, Devineni VR and Janicki JS: Prevention of angiotensin II induced myocyte necrosis and coronary vascular damage by lisinopril and losartan in the rat. Cardiovasc Res 29: 543-548, 1995 .

24. Drogat B, Auguste P, Nguyen DT, Bouchecareilh M, Pineau R, Nalbantoglu J, Kaufman RJ, ChevetE, Bikfalvi A and Moenner M: IRE1 signaling is essential for ischemia-induced vascular endothelial growth factor-A expression and contributes to angiogenesis and tumor growth in vivo. Cancer Res 67: 6700-6707, 2007.

25. Wang X, Bai YP, Hong D, Gao HC, Li LF, Li CC, Zhu LP, Sun Q and Zhang GG: Ang II induces capillary formation from endothelial cells via the AT1R-dependent inositol requiring enzyme 1 pathway. Biochem Biophys Res Commun 434: 552-558, 2013.

26. Hu P, Han Z, Couvillon AD, Kaufman RJ and Exton JH: Autocrine tumor necrosis factor alpha links endoplasmic reticulum stress to the membrane death receptor pathway through IRElalpha-mediated NF-kappaB activation and downregulation of TRAF2 expression. Mol Cell Biol 26: 3071-3084, 2006.

27. Kaneko M, Niinuma Y and Nomura Y: Activation signal of nuclear factor-kappa B in response to endoplasmic reticulum stress is transduced via IRE1 and tumor necrosis factor receptorassociated factor 2. Biol Pharm Bull 26: 931-935, 2003. 
28. Wu L, Wang D, Xiao Y, Zhou X, Wang L, Chen B, Li Q, Guo X and Huang Q: Endoplasmic reticulum stress plays a role in the advanced glycation end product-induced inflammatory response in endothelial cells. Life Sci 110: 44-51, 2014.

29. Urano F, Wang X, Bertolotti A, Zhang Y, Chung P, Harding HP and Ron D: Coupling of stress in the ER to activation of JNK protein kinases by transmembrane protein kinase IRE1. Science 287: 664-666, 2000.

30. Miyazaki-Anzai S, Masuda M, Demos-Davies KM, Keenan AL, Saunders SJ, Masuda R, Jablonski K, Cavasin MA, Kendrick J, Chonchol M, et al: Endoplasmic reticulum stress effector CCAAT/enhancer-binding protein homologous protein (CHOP) regulates chronic kidney disease-induced vascular calcification. J Am Heart Assoc 3: e000949, 2014.

31. Galkina $E$ and Ley K: Immune and inflammatory mechanisms of atherosclerosis (*). Annu Rev Immunol 27: 165-197, 2009.

32. Libby P: Inflammation in atherosclerosis. Nature 420: 868-874, 2002.

33. Guo F, Chen XL, Wang F, Liang X, Sun YX and Wang YJ: Role of angiotensin II type 1 receptor in angiotensin II-induced cytokine production in macrophages. J Interferon Cytokine Res 31: 351-361, 2011

34. Xanthoulea S, Curfs DM, Hofker MH and de Winther MP: Nuclear factor kappa B signaling in macrophage function and atherogenesis. Curr Opin Lipidol 16: 536-542, 2005.
35. de Winther MPJ, Kanters E, Kraal G and Hofker MH: Nuclear factor kappaB signaling in atherogenesis. Arterioscler Thromb Vasc Biol 25: 904-914, 2005.

36. Martinon F, Chen X, Lee AH and Glimcher LH: TLR activation of the transcription factor XBP1 regulates innate immune responses in macrophages. Nat Immunol 11: 411-418, 2010.

37. Takeya M, Yoshimura T, Leonard EJ and Takahashi K: Detection of monocyte chemoattractant protein-1 in human atherosclerotic lesions by an anti-monocyte chemoattractant protein-1 monoclonal antibody. Hum Pathol 24: 534-539, 1993.

38. Ylä-Herttuala S, Lipton BA, Rosenfeld ME, Särkioja T, Yoshimura T,Leonard EJ, Witztum JL and Steinberg D: Expression of monocyte chemoattractant protein 1 in macrophage-rich areas of human and rabbit atherosclerotic lesions. Proc Natl Acad Sci USA 88: 5252-5256, 1991.

39. Boring L, Gosling J, Cleary M and Charo IF: Decreased lesion formation in CCR $2^{-/-}$mice reveals a role for chemokines in the initiation of atherosclerosis. Nature 394: 894-897, 1998

40. Gu L, Okada Y, Clinton SK, Gerard C, Sukhova GK, Libby P and Rollins BJ: Absence of monocyte chemoattractant protein-1 reduces atherosclerosis in low density lipoprotein receptor-deficient mice. Mol Cell 2: 275-281, 1998. 